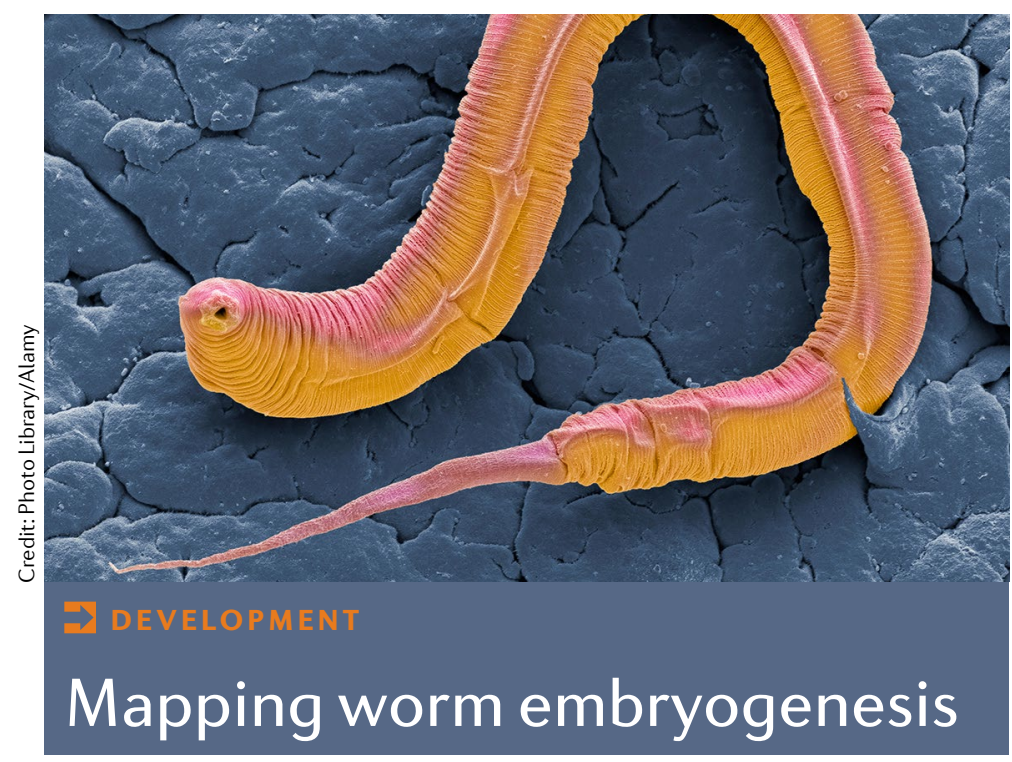

Resolving the link between temporal gene expression dynamics and cell fate decisions is essential to understand organismal development. Developmental trajectories can be reconstructed from single-cell RNA sequencing (scRNA-seq) data, but some lineage branches can be ambiguous or misleading. Now, a study in Science has leveraged the invariant cell lineage of Caenorhabditis elegans to generate a lineage-resolved single-cell atlas of C. elegans embryogenesis.

scRNA-seq data were generated for 86,024 single cells from developing C. elegans embryos. The developmental stage (or 'embryo time') of each cell was estimated by comparing its expression profile to time-resolved whole-embryo RNA-seq data. The single-cell data were then visualized with the uniform manifold approximation and projection (UMAP) algorithm and clustered using the Louvrain algorithm. Clusters were subsequently annotated for cell type based on marker gene expression. Recursive generation of 'sub-UMAPs' for subsets of cells stratified by cell type or embryo time enabled better resolution of cell or lineage identities. Overall, $93 \%$ of cells were assigned a cell type or cell lineage, and 1,068 of the 1,228 branches of the C. elegans embryonic lineage that do not lead to programmed cell death were represented in the final atlas.

Analysis of bifurcating trajectories, in which a parent cell gives rise to two different daughter cell types, suggests that each branchpoint decision hinges upon daughter-specific expression of 3-4 transcription factors (TFs). Interestingly, multi-lineage priming, in which both sets of daughter-specific TFs are expressed by the parent cell, seems to be common and pervasive during C. elegans embryogenesis.

Other UMAP trajectories would not have been easily resolved based solely on single-cell transcriptome data and highlight the limitations of such approaches. Examples include convergent trajectories, in which the gene expression patterns of cell lineages with similar or identical terminal cell fates converge; and discontinuous trajectories, in which the gene expression profile changes abruptly at the final cell division when the progenitor cell becomes terminally differentiated. Taken together, these findings indicate that scRNA-seq data alone are unlikely to be sufficient to resolve developmental trajectories for other organisms: integration with lineage tracing methods and improved computational tools will be required.

Moving forwards, data sets for missing developmental stages will be needed to complete the $C$. elegans atlas. However, integrated multi-omic analyses of mutant embryos are likely to be required to elucidate the molecular mechanisms underlying cell fate decisions.

Dorothy Clyde

ORIGINAL ARTICLE Packer, J. S. et al. A lineageat single-cell resolution. Science https://doi.org/ 10.1126/science.aax1971 (2019) resolved molecular atlas of C. elegans embryogenesis
METAGENOMICS

\section{Early-life antibiotic use and gut microbiota}

The majority of preterm infants with very low birth weight who are admitted to neonatal intensive care units (NICUs) in the USA receive antimicrobial therapy, and any resulting antibiotic-induced perturbation of the gut microbiota was thought to resolve with time. Now, a study in Nature Microbiology reports metagenomic signatures of early-life antibiotic treatment

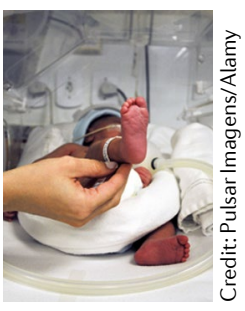
and hospitalization in preterm infants that persist long-term.

Whole-metagenome shotgun sequencing was performed on 437 stool samples, obtained from 58 infants over the first 21 months of life. The cohort included 17 antibiotic-naive, healthy infants born near term and 41 preterm infants who received a single course of antibiotics $(n=9)$ or prolonged antibiotics therapy $(n=32)$.

The species composition of the preterm infant gut microbiota was found to cluster by both gestational age at birth and treatment status. Early-life antibiotic treatment was associated with decreases in both species richness and species diversity. Antibiotics-treated infants exhibited younger predicted microbiota 'ages' than chronological age across several stages of development, which is indicative of a disruption in microbiota development. However, by 12-15 months of infant age, computed microbiota-for-age $z$-scores of antibiotics-treated preterm infants resembled those of antibiotic-naive, near-term infants, suggesting partial recovery of microbiota immaturity following hospital discharge.

The team determined the antibiotic resistome of 217 preterm and near-term infants by functional metagenomic analysis, sequencing resistance-conferring metagenomic inserts and assembling 874 unique antibiotic resistance genes (ARGs). Antibiotics-specific enrichment of ARGs was observed in preterm, treated infants, with Enterobacteriaceae and uncultured lineages identified as the likely source of ARGs. Multidrugresistant Enterobacteriaceae lineages of on average $>99.997 \%$ nucleotide identity were measured in cultured isolates of preterm infants' stool samples taken 8-10 months apart, that is, before and after discharge from the NICU. This finding suggests that antibiotic treatment, hospitalization and/or premature birth have long-term effects on the gut microbiome.

Finally, the authors devised a machine learning model that was able to distinguish infants born preterm who received early-life antibiotics from unhospitalized, near-term infants based on the species and ARGs present in the gut microbiota following hospital discharge (or at concordant time points in untreated infants). The role this persistent metagenomic signature of early-life antibiotic treatment and hospitalization has in chronic diseases of preterm infants remains uncertain, but this study emphasizes the need for alternatives to broad-spectrum antibiotics in this vulnerable patient population.

Linda Koch

ORIGINAL ARTICLE Gasparrini, A. J. et al. Persistent metagenomic signatures of early-life hospitalization and antibiotic treatment in the infant gut microbiota and resistome. Nat. Microbiol. https://doi.org/10.1038/s41564-019-0550-2 (2019) RELATED ARTICLE Boolchandani, M., D'Souza, A. W. \& Dantas, G. Sequencing-based methods and resources to study antimicrobial resistance. Nat. Rev. Genet. 20, 356-370 (2019) 